\title{
Impacto en la calidad de vida del tratamiento de disfonía espasmódica aductora con toxina botulínica A
}

\section{Impact on the quality of life of the treatment of adductor spasmodic dysphonia with botulinum toxin A}

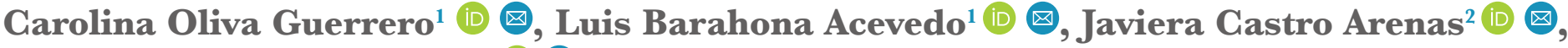 \\ Christian Olavarría Leiva ${ }^{1,2}$ (1) \\ ${ }^{1}$ Hospital Clínico Universidad de Chile; Santiago; Chile. \\ ${ }^{2}$ Universidad de Chile; Santiago; Chile.
}

\section{Correspondencia}

Christian Olavarría Leiva.

Email: christian.olavarria@gmail.com

Gitar así

Oliva Guerrero, Carolina; Barahona

Acevedo, Luis; Castro Arenas, Javiera;

Olavarría Leiva, Christian. (2021). Impacto

en la calidad de vida del tratamiento de disfonía espasmódica aductora con toxina botulínica A. Revista de Investigación e Innovación en Ciencias de la Salud. 3(2),

24-34. https://doi.org/10.46634/riics.66

Recibido: 16/05/2021

Revisado: 11/09/2021

Aceptado: 04/10/2021

\section{Invited editor}

Carlos Manzano Aquiahuatl, MD, MSc. (iD)

Editor en jefe

Jorge Mauricio Cuartas Arias, Ph.D. ID

Coeditor

Fraidy-Alonso Alzate-Pamplona, MSc.

Gopyright $($ ) 2021. Fundación Universitaria María Cano. La Revista de Investigación e Innovación en Ciencias de la Salud proporciona acceso abierto a todo su contenido bajo los términos de la licencia Creative Commons Attribution-NonCommercial-NoDerivatives 4.0 International (CG BY-NG-ND 4.0).

Declaración de intereses

Los autores han declarado que no hay

conflicto de intereses.

\section{Resumen}

Introducción. La distonía laríngea o disfonía espasmódica se caracteriza por contracciones involuntarias de los músculos laríngeos internos que se desencadenan al hablar, siendo la forma aductora la más frecuente. La inyección de toxina botulínica es el manejo de elección. Para evaluar la respuesta a la terapia existen varios instrumentos validados, uno de ellos es el cuestionario Voice Handicap Index-10 (VHI-10). El objetivo de este estudio es caracterizar a los pacientes con disfonía espasmódica aductora y evaluar el impacto de la toxina en su calidad de vida.

Método. Éste se centró en un estudio retrospectivo descriptivo en pacientes adultos con diagnóstico de disfonía espasmódica aductora tratados con toxina botulínica A, en el Hospital Clínico Universidad de Chile (HCUG), en el periodo comprendido entre 2013 y 2021. El mismo permitió la obtención de los datos epidemiológicos de los pacientes, a quienes se les solicitó responder la encuesta VHI-10 previo y posterior a un mes de cada inyección de la toxina.

Resultados. Se incluyeron 55 pacientes (218 procedimientos). La dosis promedio utilizada fue de 9,18 UI con un intervalo promedio de 7,4 meses. El puntaje VHI10 promedio en la evaluación inicial fue de 29,4 y posinfiltración de 14,96, siendo esta diferencia significativa $(\mathrm{p}<0,000001)$. En nuestra serie casi un tercio tiene asociada alguna patología neurológica, y se reportó un 3,67\% de complicaciones leves y transitorias.

Conclusión. La disfonía espasmódica aductora tiene un gran impacto en la calidad de vida de los pacientes, que se reduce significativamente mediante la inyección de toxina botulínica A, procedimiento que ha demostrado ser seguro y eficaz.

\section{Palabras clave}

Distonía; disfonía; toxina botulínica; disfonía espasmódica; distonía laríngea; disfonía espasmódica aductora; vocología. 
Disponibilidad de datos

Todos los datos relevantes se encuentran en el artículo. Para mayor información, comunicarse con el autor de correspondencia.

\section{Financiamiento}

Ninguno. Esta investigación no recibió ninguna subvención específica de agencias de financiamiento en los sectores público, comercial o sin fines de lucro.

\section{Descargo de responsabilidad}

El contenido de este artículo es responsabilidad exclusiva de los autores y no representa una opinión oficial de sus instituciones ni de la Revista de Investigación e Innovación en Ciencias de la Salud.

\section{Contribución de los autores} Carolina Oliva Guerrero: conceptualización, curación de datos, análisis formal, investigación, metodología, supervisión, validación, visualización, escritura: borrador original y escritura: revisión y edición.

Luis Barahona Acevedo: conceptualización, curación de datos, análisis formal, investigación, metodología, validación, visualización y escritura: borrador original.

Javiera Castro Arenas conceptualización y curación de datos. Christian Olavarría Leiva: conceptualización, investigación, metodología, supervisión, validación y visualización

\section{Abstract}

Introduction. Laryngeal dystonia or spasmodic dysphonia is characterized by involuntary contractions of internal laryngeal muscles that are triggered when speaking, being the adductor form the most frequent. Botulinum toxin injection is the management of choice. There are several validated instruments to assess response to therapy, one of them is the Voice Handicap Index-10 (VHI-10) questionnaire. The objective of this study is to characterize patients with adductor spasmodic dysphonia and evaluate the impact of the toxin in their quality of life.

Method. A descriptive retrospective study was carried out in adult patients with a diagnosis of adductor spasmodic dysphonia treated with botulinum toxin A, between 2013-2021 at the Hospital Clínico Universidad de Chile. The epidemiological data of the patients and the VHI-10 survey were obtained before and after one month of each toxin injection was requested.

Results. 55 patients (218 procedures) were included. The average dose used was 9.18 IU with an average interval of 7.4 months. The average VHI-10 score in the initial evaluation was 29.4 and post-infiltration was 14.96, being this difference significant ( $\mathrm{p}<0.000001)$. In our series, almost a third had an associated neurological pathology, and $3.67 \%$ of mild and transitory complications were reported.

Conclusion. Adductor spasmodic dysphonia has a great impact on the quality of life of patients, which is significantly reduced by injecting botulinum toxin $\mathrm{A}$, a procedure that has been shown to be safe and effective.

\section{Key words}

Dystonia; dysphonia; botulinum toxin; spasmodic dysphonia; laryngeal dystonia; adductor spasmodic dysphonia; vocology.

\section{Introducción}

La distonía laríngea o disfonía espasmódica es una distonía caracterizada por contracciones involuntarias de los músculos laríngeos internos [1]. Corresponde a una alteración de una tarea específica, es decir, se desencadena al hablar y afecta la fluencia de la voz. Fue descrita por primera vez en 1871 por Ludwig Traube como una "forma espástica de ronquera nerviosa" [2], y durante muchos años tratada como etiología psicológica o psiquiátrica.

Esta patología es causada por una anormalidad en el procesamiento motor del habla a nivel central y aunque se desconoce con exactitud su fisiopatología, se han demostrado alteraciones estructurales en la organización cerebral de los pacientes que la padecen, como una disfunción de la corteza sensoriomotora y la reducción focal de la densidad axonal y mielina a lo largo del tracto corticobulbar/corticoespinal [3]. A través de una resonancia magnética funcional se ha identificado un mayor grado de activación en las regiones corticales del cerebro responsables del control de la producción de voz, tanto en tareas sintomáticas como asintomáticas; mientras que el grado de activación dentro de las estructuras subcorticales (ganglios basales, tálamo y cerebelo) aumentó solo durante el habla sintomática, pero disminuyó durante las tareas laríngeas asintomáticas $[4,5]$. 
Se estima que afecta a 1:100.000 personas. Sin embargo, la dificultad en su diagnóstico, incluso para especialistas, podría provocar una subestimación del valor real. Más del $65 \%$ de los pacientes son mujeres, con una edad promedio de inicio a los 45 años [6]. Se asocia con frecuencia a comorbilidad neurológica como temblor vocal en el $25 \%$ de los casos, y en un 7-11\% a otras distonías.

Su diagnóstico es clínico, mediante la evaluación del habla del paciente, que se caracteriza por quiebres vocales irregulares e incontrolables que afectan la fluencia del habla, especialmente puede evaluarse en la emisión sostenida de una vocal, lo que permite apreciar los espasmos irregulares que determinan una voz entrecortada. No existen test diagnósticos específicos para la distonía laríngea y es necesaria la experiencia para la diferenciación con otras patologías vocales tales como temblor vocal y disfonía músculo tensional [1]. Se requiere la visualización de la laringe de forma endoscópica para confirmar los espasmos laríngeos durante la fonación y excluir otras patologías que interfieran con la producción del habla. Además, pueden utilizarse encuestas perceptuales de la voz, mediciones acústicas y la electromiografía laríngea. Empero, ninguna de estas ha mostrado validez para diferenciar la distonía laríngea de otras alteraciones vocales [7].

Existen dos tipos principales de distonía laríngea: la aductora, caracterizada por espasmos de hiperaducción que interrumpen la fonación de las cuerdas vocales, que produce una voz entrecortada, áspera y con quiebres vocales; y la abductora, con prolongadas consonantes mudas antes del inicio de las vocales que determinan una voz hipofónica, forzada y susurrada [8]. Además, las distonías pueden clasificarse en focales, segmentarias o generalizadas, de acuerdo con la cantidad de estructuras comprometidas [9].

Si bien a la fecha no existe método curativo efectivo, la inyección con toxina botulínica es el tratamiento más usado en pacientes con disfonía espasmódica, teniendo un efecto estimado de tres a cuatro meses [10], aunque podría extenderse hasta seis [11]. Existen varios y validados instrumentos para evaluar la respuesta a la terapia que demuestran reducciones significativas del impacto de la enfermedad, aunque sin lograr normalizar el puntaje [10]. Entre estos cuestionarios se encuentra el índice de discapacidad vocal (VHI, Voice Handicap Index por sus siglas en inglés) y su versión abreviada VHI-10, que ha demostrado tener similar potencia para cuantificar el impacto percibido en la propia función vocal, capacidad física en relación con ella y en las emociones provocadas por la disfonía [12,13].

El objetivo del presente estudio es describir las características clínicas y epidemiológicas de los pacientes con disfonía espasmódica aductora que se mantienen en tratamiento con inyecciones laríngeas de toxina botulínica A y determinar la efectividad de la intervención en cuanto al impacto en la calidad de vida relacionada con la voz.

\section{Materiales y método}

Los pacientes con alguna patología vocal que requieren tratamiento con toxina botulínica A son citados a un policlínico exclusivo para este propósito, el cual se realiza de forma mensual. Agrupar a estos pacientes permite la sistematización de la atención y abaratar costos para ellos ya que, debido a las bajas dosis empleadas, un mismo frasco de la toxina puede ser utilizado por distintos pacientes.

El tratamiento se realiza de forma ambulatoria, de acuerdo con el siguiente procedimiento: El frasco de 100 UI (Reage 100 UI, Abbott) se lleva a una dilución 1 UI de en 0,01 ml de agua bidestilada. La infiltración se realiza a través de una jeringa tuberculina, lo que permite que 
cada graduación de la jeringa corresponda a 1 UI. La administración se realiza a través de una inyección percutánea, en el músculo tiroaritonoídeo, bajo visión endoscópica. La punción generalmente es realizada a través de la membrana cricotiroídea, pero también puede ser sobre la escotadura tiroídea en caso de osificación de la membrana.

Los datos epidemiológicos de los pacientes se obtuvieron, inicialmente, a través de una evaluación vocal y, posteriormente, a un mes de la inyección de toxina, utilizando la encuesta VHI-10 abreviada (Tabla 1).

\begin{tabular}{l|c|c|c|c|c}
\multicolumn{4}{c}{ Tabla 1. Indfee de Calidad Vocal A breviado (VII-10) } \\
\hline La gente me oye con dificultad debido a mi voz & 0 & 1 & 2 & 3 & 4 \\
\hline La gente no me entiende en sitios ruidosos & 0 & 1 & 2 & 3 & 4 \\
\hline Mis problemas con la voz alteran mi vida personal y social & 0 & 1 & 2 & 3 & 4 \\
\hline Me siento desplazado de las conversaciones por mi voz & 0 & 1 & 2 & 3 & 4 \\
\hline Mi problema con la voz afecta al rendimiento laboral & 0 & 1 & 2 & 3 & 4 \\
\hline Siento que necesito tensar la garganta para producir la voz & 0 & 1 & 2 & 3 & 4 \\
\hline La calidad de mi voz es impredecible & 0 & 1 & 2 & 3 & 4 \\
\hline Mi voz me molesta & 0 & 1 & 2 & 3 & 4 \\
\hline Mi voz me hace sentir cierta minusvalía & 0 & 1 & 2 & 3 & 4 \\
\hline La gente me pregunta: ¿Qué te pasa con la voz? & 0 & 1 & 2 & 3 & 4
\end{tabular}

Nota. Adaptado de Núñez-Batalla et al., 2007 [13].

Para el análisis estadístico se utilizó el programa SPSS, versión 20. El p value fue considerado significativo menor o igual a 0,05 , y para determinar la respuesta mediante el cuestionario VHI-10 se utilizó la prueba $t$ de Student. Los gráficos fueron creados con Prism versión 9. Este estudio se encuentra aprobado por el comité del Hospital Clínico de la Universidad de Chile.

\section{Resultados}

El estudio incluyó como muestra un total de 55 pacientes, de los cuales 39 acudieron más de una vez al procedimiento. El 80 \% fue de sexo femenino (44) y la edad promedio fue de 58,7 años, con un rango de 19 a 89 años. En la Tabla 2 se resumen los datos obtenidos.

\begin{tabular}{|c|c|c|c|c|}
\hline & Total pacientes & Mujeres & Hombres & $p$ value \\
\hline $\mathrm{N}^{\circ}(\%)$ & $55(100 \%)$ & $44(80 \%)$ & $11(20 \%)$ & - \\
\hline Edad promedio & 58,7 & 61,7 & 49,3 & $p=0,033$ \\
\hline$\%$ Comorbilidades $\left(n^{\circ}\right)$ & $27,27 \%(15)$ & $27,27 \%(12)$ & $27,27 \%(3)$ & $p=0,99$ \\
\hline Total infiltraciones & 218 & 160 & 58 & - \\
\hline Dosis promedio UI & 9,18 & 8,8 & 10,7 & $p=0,2592$ \\
\hline Intervalo promedio (meses) & $7,4 \mathrm{~m}$ & $6,6 \mathrm{~m}$ & $10,2 \mathrm{~m}$ & $p=0,0183$ \\
\hline VHI-10 previo & 29,4 & 29,7 & 30,06 & $p=0,9873$ \\
\hline VHI-10 posterior & 14,96 & 14,85 & 13,77 & $p=0,7428$ \\
\hline$\%$ Efectos adversos $\left(n^{\circ}\right)$ & $3,67 \%(8)$ & $4,37 \%(7)$ & $1,72 \%(1)$ & $p=0,6847$ \\
\hline
\end{tabular}


En total, se realizaron 218 procedimientos en el periodo de estudio, 158 de ellos por el lado izquierdo del paciente (72,5 \% de las infiltraciones), 38 bilaterales y solo 22 fueron exclusivamente del lado derecho.

La dosis promedio utilizada por procedimiento fue de 9,18 UI: 10,7 UI aplicadas a hombres, versus 8,8 UI en mujeres. Sin embargo, esta diferencia no fue significativa. A su vez, el intervalo promedio fue de 7,4 meses entre cada dosis, siendo significativamente mayor en hombres que en mujeres, con 10,2 vs 6,6 meses, respectivamente $(\mathrm{p}=0,0183, t$ student $)$.

El índice VHI-10 promedio, al momento de la evaluación inicial, fue de 29,4 + 10,3 y posinfiltración fue de 14,96 + 12,48, siendo esta diferencia estadísticamente significativa utilizando la prueba $t$ de Student ( $\mathrm{p}<0,000001$ ) (Figura 1). No existieron diferencias al separar en grupos por sexo ni tampoco por edad de los pacientes. El VHI-10 es capaz de evaluar el impacto de la patología vocal en el área funcional, física y emocional de los pacientes [13], en nuestros datos analizados no existieron diferencias significativas entre estas esferas $(p=0,36$, prueba $x i$-cuadrado).

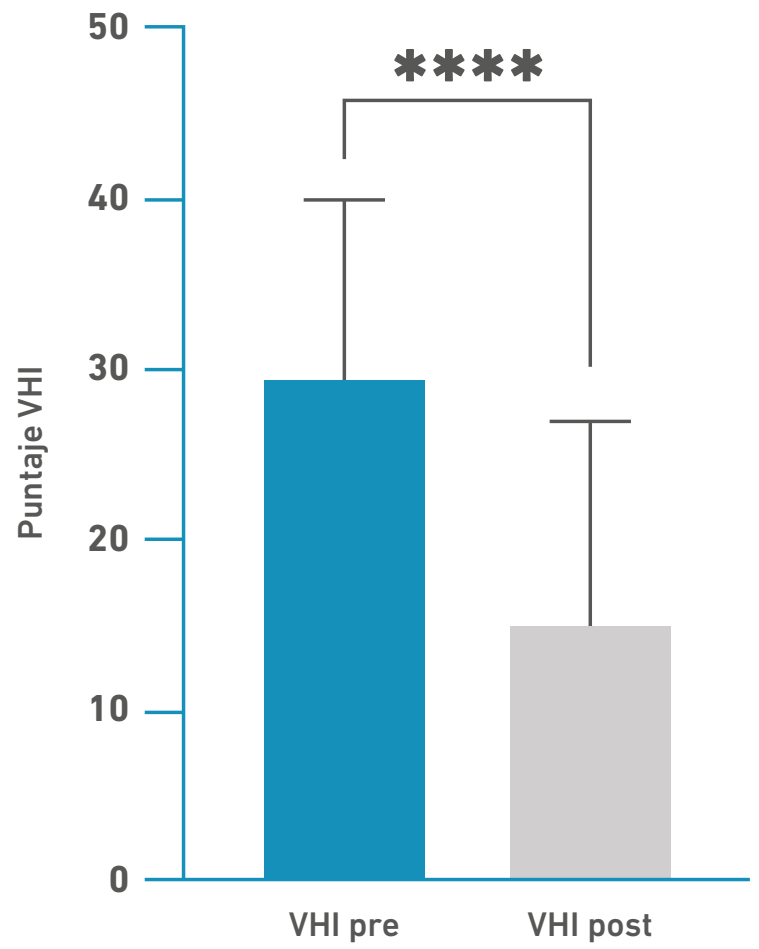

Figura 1. Respuesta a toxina botulínica A medido a través de VHI-10 inicial y posterior, a un mes de inyección. $(p<0,0001, t$ student $)$

En la Figura 2 podemos observar que los pacientes que tuvieron mayor número de infiltraciones poseen una tendencia a un menor puntaje VHI-10; es decir, una relación inversa entre cantidad de infiltraciones por paciente e índice de discapacidad vocal. Sin embargo, este dato no fue estadísticamente significativo $(p=0,1604$, Anova test $)$. 


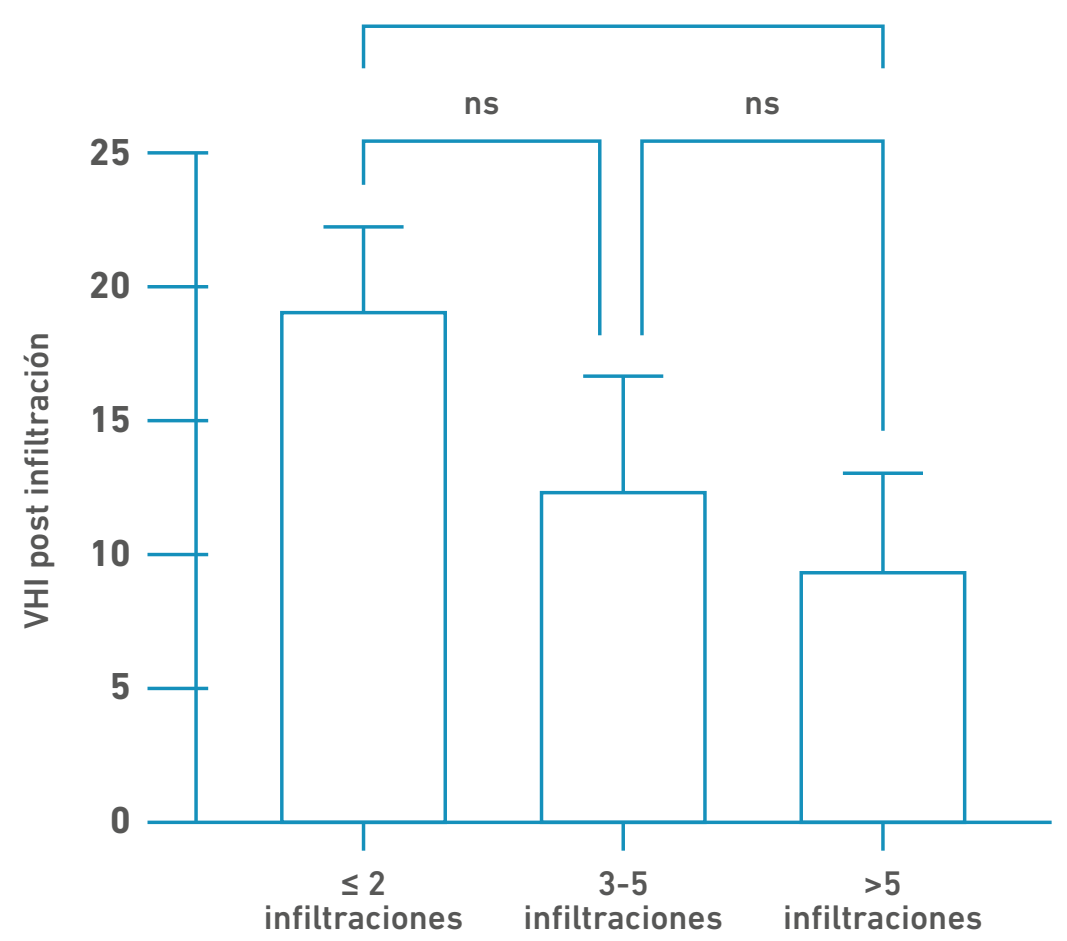

Figura 2. VHI post infiltración según número de infiltraciones ( $p=0,1604$, Anova test)

Como se mencionó previamente, es frecuente que los pacientes con distonía laríngea presenten alguna comorbilidad neurológica. En nuestra serie de casos, 15 de ellos tenía asociada alguna patología neurológica $(27,27 \%), 8$ de ellos temblor vocal $(14,5 \%), 6$ otras distonías $(10,9 \%)$ y 1 paciente con enfermedad de Parkinson (Figura 3).

De los 218 procedimientos, solo ocho de ellos tuvieron algún evento adverso (3,67 \% de complicaciones), cuatro pacientes presentaron disfagia leve a moderada, tres pacientes con voz soplada de más de dos semanas de evolución y una paciente presentó una reacción cutánea alérgica al contacto con el látex de los guantes utilizados en el procedimiento.

\section{Discusión}

La disfonía espasmódica aductora es una enfermedad infrecuente, que afecta en gran medida la calidad de vida de los pacientes que padecen esta condición [10]. De acuerdo con las series más extensas publicadas en la literatura, afecta principalmente a mujeres en la edad media de la vida, consistente con nuestra casuística en que el $80 \%$ corresponden a mujeres [14-17].

En ocasiones se puede observar asociado a otras comorbilidades neurológicas, entre ellas temblor vocal, en un 17.6-52.6 \%, y otras distonías, en un 5.2-37.5 \% [15,18-20]. Nuestra casuística está en consonancia con lo descrito en la literatura, reportando que un $14.5 \%$ de nuestros pacientes tienen temblor vocal asociado y $10.9 \%$ otras distonías, principalmente cervical y oromandibular. 


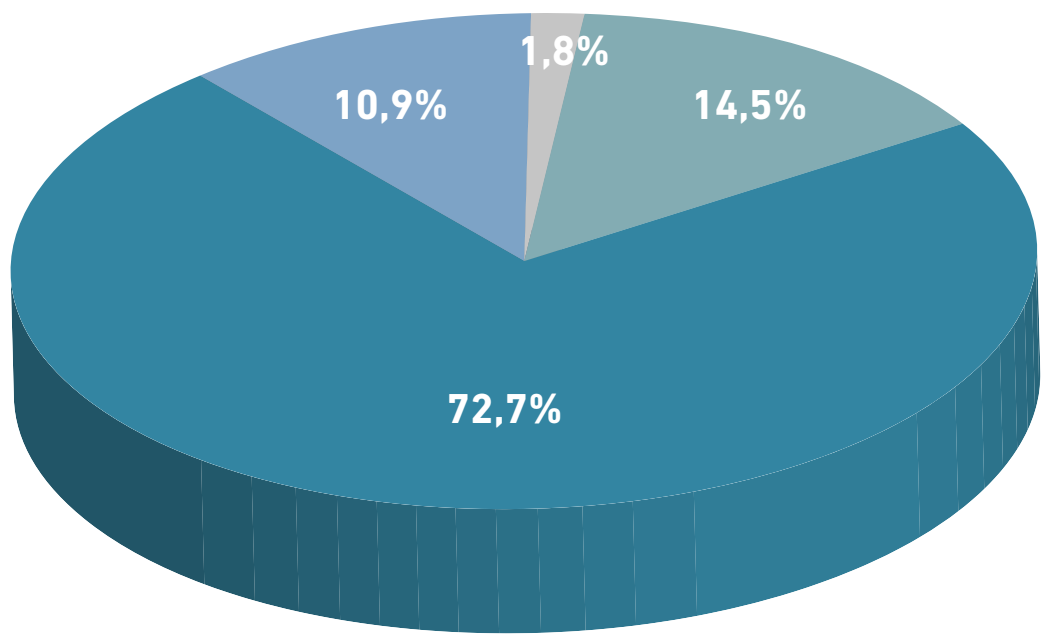

Temblor vocal $(14,5 \%)$

Sin comorbilidad $(72,7 \%)$

Otras distonías (10,9\%)

Enfermedad de Parkinson (1,8\%)

Figura 3. Presencia de comorbilidad neurológica en pacientes con distonía laríngea.

La administración de toxina botulínica inyectada en el músculo tiroaritonoideo es el pilar del tratamiento de la disfonía espasmódica en la actualidad [16,17]. De acuerdo con una encuesta reciente, respondida por laringólogos americanos, la elección de la dosis inicial está basada en el balance de deseo y necesidades de los pacientes y de la fragilidad y el riesgo de aspiración. La gran mayoría de los expertos inicia con inyección bilateral, con un rango de 0,25 a 5 unidades [21], de las cuales aplican 1,25 unidades por lado.

Se ha determinado que la dosis de toxina botulínica alcanzada en la titulación sería mayor en pacientes con mayor índice de masa corporal y con mejor salud global, sin diferencias significativas respecto a la edad ni al género [21,22]. El factor socioeconómico si bien podría afectar el número y frecuencia de las inyecciones, no tendría relación con la dosis requerida [22]. Se ha reportado que, con las inyecciones seriadas, la dosis tendería a disminuir progresivamente $[17,23]$.

En el HCUC, en general, se inicia con cuatro unidades, similar a otras experiencias reportadas [20,23], y se titula la dosis de acuerdo con la evolución de cada paciente. Existe una tendencia a aplicarse mayores dosis en hombres que en mujeres, lo que difiere con lo descrito en la mayoría de la literatura, lo que podría explicarse por una menor adherencia al tratamiento en los hombres, sugerido por el mayor intervalo entre dosis.

En una revisión sistemática y de metaanálisis reciente, se ha visto que tanto la inyección unilateral como bilateral se asocia a efectos significativamente positivos [10]. En el centro clínico de la Universidad de Chile, la mayoría de los pacientes han recibido inyección unilateral 
en el músculo tiroaritenoideo izquierdo, principalmente por motivos ergonómicos de quienes administran la inyección.

$\mathrm{Al}$ igual que en la mayoría de los lugares en donde se aplican inyecciones de toxina botulínica laríngea [24], en el HCUG se extraen múltiples dosis por vial para pacientes distintos, los que son citados en un único día. Según un análisis reciente, se ha estimado que el costo anual de pacientes con uso único del vial sería alrededor de USD 2050, lo que representa un $1118 \%$ mayor al costo estimado en el uso de múltiples dosis de aproximadamente USD 168, lo que no comprometería ni la seguridad ni eficacia del tratamiento [25].

Existen distintos instrumentos para valorar el impacto en la calidad de vida de pacientes con patología de la voz. La primera encuesta en ser publicada, una de las más utilizadas en la actualidad, fue desarrollada por Jacobson et al. y corresponde al "Índice de Discapacidad Vocal" (VHI) [26]. La encuesta consta de treinta ítems y evalúa las consecuencias de los trastornos vocales relacionadas con las esferas emocionales, funcionales y físicas. Posteriormente, Rosen et al. publicaron una versión abreviada denominada VHI-10, que se ocupa únicamente de las diez preguntas más robustas de la encuesta original, cuya aplicación no mostró diferencias estadísticas respecto a la versión extendida y poseer una alta correlación independiente del diagnóstico, proponiéndose su uso debido a su rápida aplicabilidad [12]. Tanto el VHI como el VHI-10 cuentan con traducciones al castellano, habiéndose probado su uso por su validez y fiabilidad [13].

Particularmente, respecto al uso de encuestas de calidad de vida en la evaluación del tratamiento con toxina botulínica en pacientes con distonía laríngea, se ha reportado que el VHI, VHI-10 y la encuesta V-RQOL (por sus siglas en inglés Voice-Related Quality of Life) tienen una alta correlación y reflejan cambios significativos de la intervención, por lo que algunos autores sugieren que el médico realice la encuesta que prefiera [27]. Esto también ha sido observado en otro estudio donde VHI y VHI-10 parecieran tener sensibilidad similar en pacientes con disfonía espasmódica [28].

Los resultados obtenidos en el centro clínico del HCUG son consistentes con otros estudios que han utilizado la encuesta VHI-10 para evaluar los efectos de la inyección de toxina botulínica en pacientes con disfonía espasmódica aductora, variando desde un puntaje previo de 20,86-27,86 a un puntaje de 10,86-20,86 en plazos variables posteriores al procedimiento (Tabla 3) [27-31]. Sin embargo, es necesario complementar estos resultados aplicando el laboratorio de voz en estos pacientes.

\begin{tabular}{|c|c|c|c|c|}
\hline Estudio & $\mathrm{N}$ & $\begin{array}{c}\text { VHI-10 previo } \\
\text { Promedio } \\
\text { [Desviación estándar] }\end{array}$ & $\begin{array}{c}\text { VHI-10 posterior } \\
\text { Promedio } \\
\text { [Desviación estándar] }\end{array}$ & $p$ \\
\hline Neumann et al, 2009 [28] & 12 & $24,42[4,85]$ & $16,25[5,23]$ & 0,019 \\
\hline Novakovic et al, 2011 [29] & 133 & 22,3 & 12,7 & $<0,01$ \\
\hline Morzaria et al, 2012 [27] & 37 & $24,54[7,19]$ & $15,64[6,90]$ & $<0,05$ \\
\hline Aguayo et al, 2017 [31] & 14 & $27,86[5,7]$ & $10,86[2,2]$ & $<0,0001$ \\
\hline Yiu et al, 2021 [30] & 190 & $22,58[7,09]$ & $20,86[7,24]$ & 0,042 \\
\hline
\end{tabular}


La inyección de toxina botulínica laríngea se ha asociado a efectos adversos transitorios que en general van de leves a moderados. Se ha reportado una voz soplada en hasta un $50,9 \%$, con un promedio de duración de 10 días, así como también disfagia a líquidos en hasta 14,2\%, con una duración promedio de 2 días [29]. Además, se ha descrito dolor local, prurito o equimosis relacionadas con la administración percutánea $(<1 \%)$ [14]. La administración bilateral podría producir un mejor perfil en la relación efecto y eventos adversos [32]. En los pacientes de este estudio se reportó una tasa de eventos adversos muy baja (3,67 \%), siendo leves y transitorias.

Esta casuística corresponde a la mayor publicada en literatura hispanoamericana a la fecha.

\section{Conclusión}

La disfonía espasmódica aductora tiene un gran impacto en la calidad de vida de los pacientes que padecen problemas relacionados con la voz. A través de este estudio se demostró que la inyección laríngea de toxina botulínica A es segura y efectiva para el tratamiento de esta condición, lo que se refleja en una mejoría significativa en el VHI-10 posinfiltración de los pacientes. Los datos epidemiológicos de este trabajo no difieren en general con respecto a la literatura publicada. El cuestionario VHI-10 es una herramienta confiable, de fácil aplicación, que ha demostrado ser útil para el seguimiento de estos pacientes.

\section{Referencias}

1. Whurr R, Lorch M. Review of differential diagnosis and management of spasmodic dysphonia. Curr Opin Otolaryngol Head Neck Surg. 2016;24(3):203-207. Doi: https://doi. org/10.1097/MOO.0000000000000253

2. Lorch MP, Whurr R. Tracing Spasmodic Dysphonia: The Source of Ludwig Traubes Priority. Ann Otol Rhinol Laryngol. 2016;125(8):672-676. Doi: https://doi. org/10.1177/0003489416646115

3. Mor N, Simonyan K, Blitzer A. Central voice production and pathophysiology of spasmodic dysphonia. Laryngoscope. 2018;128(1):177-183. Doi: https://doi.org/10.1002/lary.26655

4. Simonyan K, Ludlow GL. Abnormal structure-function relationship in spasmodic dysphonia. Cereb Cortex. 2012;22(2):417-425. Doi: https://doi.org/10.1093/cercor/bhr120

5. Chen M, Summers RLS, Prudente GN, et al. Transcranial magnetic stimulation and functional magnet resonance imaging evaluation of adductor spasmodic dysphonia during phonation. Brain Stimul. 2020;13(3):908-915. Doi: https://doi.org/10.1016/j. brs. 2020.03 .003

6. Hintze JM, Ludlow CL, Bansberg SF, Adler CH, Lott DG. Spasmodic Dysphonia: A Review. Part 1: Pathogenic Factors. Otolaryngol - Head Neck Surg (United States). 2017;157(4):551-557. Doi: https://doi.org/10.1177/0194599817728521

7. Ludlow CL, Domangue R, Sharma D, et al. Consensus-based attributes for identifying patients with spasmodic dysphonia and other voice disorders. JAMA Otolaryngol - Head Neck Surg. 2018;144(8):657-665. Doi: https://doi.org/10.1001/jamaoto.2018.0644

8. Hintze JM, Ludlow CL, Bansberg SF, Adler CH, Lott DG. Spasmodic Dysphonia: A Review. Part 2: Characterization of Pathophysiology. Otolaryngol - Head Neck Surg (United States). 2017;157(4):558-564. Doi: https://doi.org/10.1177/0194599817728465 
9. Brunetto B, Nazar R, Olavarría C, Gutiérrez A, del Peso G, Miranda M, Painepán JC. Experiencia con toxina botulínica en el tratamiento de la disfonía espasmódica. Rev. Otorrinolaringol. Cir. Cabeza y cuello. 2014, vol. 64, p. 105-112. Url: https://n9.cl/riics

10. Faham M, Ahmadi A, Silverman E, Harouni GG, Dabirmoghaddam P. Quality of life after botulinum toxin injection in patients with adductor spasmodic dysphonia; a systematic review and meta-analysis. Journal of Voice. 2021 Mar 1;35(2):271-83. Doi: https:// doi.org/10.1016/j.jvoice.2019.07.025

11. Faham M, Torabinezhad F, Murry T, et al. Quality of life and voice changes after a single injection in patients with ADSD over time. J Voice 2019 Sep;33(5):721-727. Doi: https:/ / doi.org/10.1016/j.jvoice.2018.04.023.

12. Rosen C, Lee AS, Osborne J, Zullo T, Murry T. Development and validation of the Voice Handicap Index-10. Laryngoscope. 2004;114:1549-1556. Doi: https://doi. org/10.1097/00005537-200409000-00009.

13. Núñez-Batalla F, Corte-Santos P, Señaris-González B, Llorente-Pendás JL, Gorriz-Gil C, Suarez-Nieto C. Adaptación y validación del índice de incapacidad vocal (VHI-30) y su versión abreviada (VHI-10) al español. Acta Otorrinolaringológica Española. 2007 Nov 1;58(9):386-92. Doi: https://doi.org/10.1016/S0001-6519(07)74954-3

14. Blitzer A. Spasmodic dysphonia and botulinum toxin: experience from the largest treatment series. European Journal of Neurology. 2010 Jul;17:28-30. Doi: https://doi. org/10.1111/j.1468-1331.2010.03047.x.

15. Patel AB, Bansberg SF, Adler CH, Lott DG, Crujido L. The Mayo Clinic Arizona spasmodic dysphonia experience: a demographic analysis of 718 patients. Annals of Otology, Rhinology \& Laryngology. 2015 Nov;124(11):859-63. Doi: https://doi. org/10.1177/0003489415588557.

16. Paddle P, Husain I, Moniz C, Turner S, Franco Jr RA. Long-term dose stability of onabotulinumtoxinA injection for adductor spasmodic dysphonia: a 19-year single institution experience. Frontiers in surgery. 2017 Nov 29;4:70. Doi: https://doi.org/10.3389/ fsurg.2017.00070. eCollection 2017.

17. French G, Bosch JD, Randall DR. Retrospective review of dosing trends in botulinum toxin injections for the treatment of adductor spasmodic dysphonia in a long-term cohort. Journal of Otolaryngology-Head \& Neck Surgery. 2020 Dec;49(1):1-4. Doi: https://doi. org/10.1186/s40463-020-0401-4.

18. Blitzer A, Brin MF, Simonyan K, Ozelius LJ, Frucht SJ. Phenomenology, genetics, and CNS network abnormalities in laryngeal dystonia: A 30-year experience. The Laryngoscope. 2018 Jan;128:S1-9. Doi: https://doi.org/10.1002/lary.27003.

19. Ozgursoy SK, Vargas ER, Heckman MG, Rutt AL. Demographics and coexisting tremor, cervical dystonia and vocal fold disorders in a group of patients with spasmodic dysphonia. Acta Otorhinolaryngologica Italica. 2020 Jun;40(3):198. doi: https://doi. org/10.14639/0392-100X-N0284.

20. Marchese MR, D'Alatri L, Bentivoglio AR, Paludetti G. OnabotulinumtoxinA for adductor spasmodic dysphonia (ADSD): functional results and the role of dosage. Toxicon. 2018 Dec 1;155:38-42. Doi: https://doi.org/10.1016/j.toxicon.2018.10.006. 
21. Shoffel-Havakuk H, Rosow DE, Lava CX, Hapner ER, Johns III MM. Common practices in botulinum toxin injection for spasmodic dysphonia treatment: a national survey. The Laryngoscope. 2019 Jul;129(7):1650-6. Doi: https://doi.org/10.1002/lary.27696.

22. Young DL, Halstead LA. Relationship of laryngeal botulinum toxin dosage to patient age, vitality, and socioeconomic issues. Journal of Voice. 2014 Sep 1;28(5):614-7.) doi: https://doi.org/10.1016/j.jvoice.2013.10.024

23. Bradley JP, Barrow EM, Hapner ER, Klein AM, Johns III MM. Botulinum toxin-a dosing trends for adductor spasmodic dysphonia at a single institution over 10 years. Journal of Voice. 2017 May 1;31(3):363-5. Doi: https://doi.org/10.1016/j.jvoice.2016.09.022.

24. McGarey Jr PO, Simpson CB, Daniero JJ. Practice Patterns and Barriers in Botulinum Toxin Injection for the Treatment of Voice Disorders. Journal of Voice. 2020 May 26;S0892-1997(20)30155-7. Doi: https://doi.org/10.1016/j.jvoice.2020.04.021.

25. Gilbert MR, Young VN, Smith LJ, Rosen CA. Multidose botulinum toxin A for intralaryngeal injection: a cost analysis. Journal of Voice. 2019 Mar 1;33(2):159-61. Doi: https://doi.org/10.1016/j.jvoice.2017.11.004.

26. Jacobson BH, Johnson A, Grywalski C, et al. The voice handicap index (VHI): development and validation. Am J Speech-Lang Pathol. 1997;6:66-70. Doi: https://doi. org/10.1044/1058-0360.0603.66

27. Morzaria S, Damrose EJ. A comparison of the VHI, VHI-10, and V-RQOL for measuring the effect of botox therapy in adductor spasmodic dysphonia. Journal of Voice. 2012 May 1;26(3):378-80. Doi: https://doi.org/10.1016/j.jvoice.2010.07.011.

28. Neumann KJ, Dejonckere PH. Voice related quality of life in spasmodic dysphonia: a detailed VHI-analysis before and after botulinum treatment. InMAVEBA 2009 Dec 14 (p. 65). Url: https://n9.cl/psg5qb

29. Novakovic D, Waters HH, D'Elia JB, Blitzer A. Botulinum toxin treatment of adductor spasmodic dysphonia: longitudinal functional outcomes. The Laryngoscope. 2011 Mar;121(3):606-12. Doi: https://doi.org/10.1002/lary.21395.

30. Yiu Y, Baylor CR, Bamer AM, Shelly S, Klein AM, Garrett CG, Pitman MJ. Validation of the Communicative Participation Item Bank as an Outcome Measure for Spasmodic Dysphonia. The Laryngoscope. 2021 Apr;131(4):859-64. Doi: https://doi.org/10.1002/ lary.28897.

31. Aguayo AM, Corrales MT, Castro GF. Índice de discapacidad vocal pre-y postaplicación de toxina botulínica en pacientes con disfonía espasmódica. Anales Médicos de la Asociación Médica del Centro Médico ABC. 2017 Jun 15;62(2):100-5. Url: https://n9.cl/7psxo

32. Dharia I, Bielamowicz S. Unilateral versus bilateral botulinum toxin injections in adductor spasmodic dysphonia in a large cohort. The Laryngoscope. 2020 Nov;130(11):265962. Doi: https://doi.org/10.1002/lary.28457. 